\title{
Twist-3 fragmentation contribution to polarized hyperon production in unpolarized proton-proton collision
}

\author{
Kenta Yabe* \\ Graduate School of Science and Technology, Niigata University, Ikarashi, Niigata 950-2181, \\ Japan \\ E-mail: K.yabe.hadron@gmail.com
}

\section{Yuji Koike}

Department of Physics, Niigata University, Ikarashi, Niigata 950-2181, Japan

E-mail: koike@nt.sc.niigata-u.ac.jp

\section{Andreas Metz}

Department of Physics, SERC, Temple University, Philadelphia, PA 19122, USA

E-mail: metzadtemple.edu

\section{Daniel Pitonyak}

Department of Physics, Lebanon Valley College, Annville, PA 17003, USA

Department of Physics, Old Dominion University, Norfolk, VA 23529, USA

E-mail: pitonyakelvc.edu

\section{Shinsuke Yoshida}

Theoretical Division, Los Alamos National Laboratory, Los Alamos, NM 87545, USA

Institute of Quantum Matter and School of Physics and Telecommunication Engineering, South

China Normal University, Guangzhou 510006, China

E-mail: shinyoshida85@gmail.com

\begin{abstract}
We discuss the production of transversely polarized hyperons in unpolarized proton-proton collisions in the framework of the collinear twist-3 factorization. We focus on the contribution from the twist-3 fragmentation functions for the final hyperon, that represent the effect of multi-parton correlations in the fragmentation process. This contribution consists of the quark fragmentation one and the gluon fragmentation one. We present the twist- 3 cross section for the former in the Leading Order (LO) with respect to the QCD coupling constant. We also present a new formalism to calculate the cross section for the latter contribution. These contributions are as important as that from the twist-3 distribution in the initial proton combined with the transversity fragmentation function for the final hyperon.
\end{abstract}

23rd International Spin Physics Symposium - SPIN2018 -

10-14 September, 2018

Ferrara, Italy

${ }^{*}$ Speaker. 


\section{Introduction}

Clarifying the origin of the large transverse polarization of produced hyperons (collectively denoted as $\Lambda$ hereafter) observed in high energy unpolarized proton-proton collision, $p p \rightarrow \Lambda^{\uparrow} X$ [1], has been a big challenge in the field of QCD spin physics. This is because the conventional twist-2 calculation (QCD parton model) leads to negligible polarization for this process. This is known as a Transverse Single Spin Asymmetries (TSSA). Other TSSAs include the spin asymmetries with respect to the initial polarization for processes such as $p^{\uparrow} p \rightarrow \pi X$ and $e p^{\uparrow} \rightarrow e \pi X$ etc.

In the collinear factorization, the SSAs appear as a twist-3 observable which represents the effect of multi-parton correlations in the initial hadrons or in the fragmentation process for the final hadron $[2,3]$ as opposed to the independent scattering off partons for the case of the twist-2 contribution. The extension of the theoretical framework to deal with this twist-3 effect is quite involved but is now available in the literature. TSSAs for $p^{\uparrow} p \rightarrow \pi X$ and $e p^{\uparrow} \rightarrow e \pi X$ have been studied in detail by using this collinear twist-3 factorization framework [4-10], but the hyperon's polarization phenomenon has not been addressed that much.

In this article, we study the twist-3 fragmentation contribution to $p p \rightarrow \Lambda^{\uparrow} X$ in the collinear twist-3 factorization. It is represented as the twist-3 quark Fragmentation Function (FF) and the twist-3 gluon FF of the hyperon. The Leading-Order (LO) cross section from the former was calculated in [11]. Here we extend the study to include the latter contribution. These contributions constitute the cross section for this process together with the previously calculated contribution from the twist-3 distribution function in the initial proton combined with the transversity FF for $\Lambda^{\uparrow}[12-14]$.

This paper is organized as follows: In Sec. 2, we discuss the twist-3 quark fragmentation contribution to $p p \rightarrow \Lambda^{\uparrow} X$. This part is a short summary of [11]. In Sec. 3, we discuss the twist-3 gluon fragmentation contribution. After summarizing the relevant gluon FFs, we present a formalism to calculate the cross section. Details of the formalism and the partonic cross section for each channel will be reported elsewhere. Sec. 4 is devoted to a brief summary.

\section{Contribution from Twist-3 quark fragmentation functions}

\subsection{Twist-3 quark fragmentation functions}

Here we summarize the twist-3 quark FFs for a transversely polarized hyperon which contribute to $p p \rightarrow \Lambda^{\uparrow} X$. They are classified as intrinsic, kinematical, and dynamical FFs [15]. Intrinsic ones are defined from the usual lightcone correlators of two quark fields as [15-17]

$$
\begin{aligned}
\Delta_{i j}(z) & =\frac{1}{N} \sum_{X} \int \frac{d \lambda}{2 \pi} e^{-i \frac{\lambda}{z}}\left\langle 0\left|\psi_{i}(0)\right| h\left(P_{h}, S_{\perp}\right) X\right\rangle\left\langle h\left(P_{h}, S_{\perp}\right) X\left|\bar{\psi}_{j}(\lambda w)\right| 0\right\rangle= \\
& =\left(\gamma_{5} \$_{\perp} \frac{\mathbb{P}_{h}}{z}\right)_{i j} H_{1}(z)+M_{h} \varepsilon^{\alpha S_{\perp} \mathrm{w} P_{h}}\left(\gamma_{\alpha}\right)_{i j} \frac{D_{T}(z)}{z}+M_{h}\left(\gamma_{5} \$_{\perp}\right)_{i j} \frac{G_{T}(z)}{z}+\cdots,
\end{aligned}
$$

where $\psi_{i}$ is a quark field with the spinor index $i$ and the color average is implied with $N=3$ the number of quark colors. In (2.1),$H_{1}(z)$ is the transversity FF, and $\left\{D_{T}(z), G_{T}(z)\right\}$ are the intrinsic twist-3 FFs. The hyperon $(\Lambda)$ is characterized by its four-momentum $P_{h}$, the transverse spin vector 
$S_{\perp}$ and the mass $M_{h}$. A lightlike vector $w^{\mu}$ satisfies $P_{h} \cdot w=1$. A gaugelink operator between the quark fields is suppressed in (2.1) for simplicity. Here and below we use the shorthand notation $\varepsilon^{\alpha S_{\perp} \mathrm{w} P_{h}} \equiv \varepsilon^{\alpha \beta \gamma \delta} S_{\perp \beta} w_{\gamma} P_{h \delta}$ with our convention for the Levi-Civita tensor $\varepsilon^{0123}=+1$.

The kinematical twist-3 FFs are obtained from the transverse derivative of the $q q$ correlator [15-17]:

$$
\begin{aligned}
\Delta_{\partial i j}^{\alpha}(z) & =\frac{1}{N} \sum_{X} \int \frac{d \lambda}{2 \pi} e^{-i \frac{\lambda}{z}}\left\langle 0\left|[\infty w, 0] \psi_{i}(0)\right| h\left(P_{h}, S_{\perp}\right) X\right\rangle\left\langle h\left(P_{h}, S_{\perp}\right) X\left|\bar{\psi}_{j}(\lambda w)[\lambda w, \infty w]\right| 0\right\rangle \overleftarrow{\partial}^{\alpha}= \\
& =-i M_{h} \varepsilon^{\alpha S_{\perp} \mathrm{w} P_{h}}\left(\boldsymbol{P}_{h}\right)_{i j} \frac{D_{1 T}^{\perp(1)}(z)}{z}+i M_{h} S_{\perp}^{\alpha}\left(\gamma_{5} \boldsymbol{P}_{h}\right)_{i j} \frac{G_{1 T}^{\perp(1)}(z)}{z}+\cdots
\end{aligned}
$$

Note that the derivative in the r.h.s. of (2.2) also acts on the gauge link $[\lambda w, \infty w] . D_{1 T}^{\perp(1)}$ and $G_{1 T}^{\perp(1)}$ are defined as real and can be written as the ${\overrightarrow{k_{\perp}}}^{2} / M_{h}^{2}$-moment of the twist-2 transverse-momentumdependent quark FFs [16, 17].

The dynamical twist-3 FFs are obtained from the correlation functions containing the gluon's field strength $F^{\alpha w} \equiv F^{\alpha \beta} w_{\beta}$ as [15],

$$
\begin{aligned}
& \Delta_{F i j}^{\alpha}\left(z, z_{1}\right)= \\
& \quad=\frac{1}{N} \sum_{X} \int \frac{d \lambda}{2 \pi} \int \frac{d \mu}{2 \pi} e^{-i \frac{\lambda}{z_{1}}} e^{-i \mu\left(\frac{1}{z}-\frac{1}{z_{1}}\right)}\left\langle 0\left|\psi_{i}(0)\right| h\left(P_{h}, S_{\perp}\right) X\right\rangle\left\langle h\left(P_{h}, S_{\perp}\right) X\left|\bar{\psi}_{j}(\lambda w) \mathrm{g} F^{\alpha w}(\mu w)\right| 0\right\rangle= \\
& \quad=M_{h} \varepsilon^{\alpha S_{\perp} \mathrm{w} P_{h}}\left(\mathbb{P}_{h}\right)_{i j} \frac{\widehat{D}_{F T}^{*}\left(z, z_{1}\right)}{z}-i M_{h} S_{\perp}^{\alpha}\left(\gamma_{5} \mathbb{P}_{h}\right)_{i j} \frac{\widehat{G}_{F T}^{*}\left(z, z_{1}\right)}{z}+\cdots
\end{aligned}
$$

We note that the dynamical FFs $\widehat{D}_{F T}$ and $\widehat{G}_{F T}$ are complex functions: their real parts are naïvely $T$-even, while the imaginary parts are naïvely $T$-odd. The replacement $g F^{\alpha w} \rightarrow D^{\alpha}$ on the 1.h.s. of (2.3) defines other twist-3 FFs, but they can be related to the above FFs. For $p p \rightarrow \Lambda^{\uparrow} X$, the naïvely $T$-odd functions $D_{T}, D_{1 T}^{\perp(1)}, \operatorname{Im} \widehat{D}_{F T}$ and $\operatorname{Im} \widehat{G}_{F T}$ appear in the twist- 3 cross section.

The above three types of FFs are not independent but obey the constraint relations which follow from the QCD equation of motion (e.o.m. relation) and the operator identities for the parton correlation functions based on the Lorentz invariance (LIR=Lorentz Invariance Relation). The e.o.m. relation relevant to our study reads [15]:

$$
\int_{z}^{\infty} \frac{d z_{1}}{z_{1}^{2}} \frac{1}{1 / z-1 / z_{1}}\left(\operatorname{Im} \widehat{D}_{F T}\left(z, z_{1}\right)-\operatorname{Im} \widehat{G}_{F T}\left(z, z_{1}\right)\right)=\frac{D_{T}(z)}{z}+D_{1 T}^{\perp(1)}(z),
$$

and the LIR is given by

$$
-\frac{2}{z} \int_{z}^{\infty} \frac{d z_{1}}{z_{1}^{2}} \frac{\operatorname{Im} \widehat{D}_{F T}\left(z, z_{1}\right)}{\left(1 / z_{1}-1 / z\right)^{2}}=\frac{D_{T}(z)}{z}+\frac{d\left(D_{1 T}^{\perp(1)}(z) / z\right)}{d(1 / z)} .
$$

These relations generally simplify the form of twist- 3 cross sections and, in particular, guarantee their frame-independence [15]. 


\subsection{Twist-3 quark fragmentation contribution to the cross section}

This section summarizes the twist-3 quark fragmentation contribution to

$$
p(p)+p\left(p^{\prime}\right) \rightarrow \Lambda^{\uparrow}\left(P_{h}, S_{\perp}\right)+X .
$$

The LO cross section was calculated in [11] following the formalism developed in [8]. It receives contributions from the three types of twist-3 FFs introduced in the previous subsection, which is diagrammatically shown in Fig. 1. For the LO cross section, using the constraint relations (2.4) and (2.5), we found that the result can be written in the frame-independent form as

$$
\begin{aligned}
P_{h}^{0} \frac{d \sigma\left(p, p^{\prime}, P_{h}, S_{\perp}\right)}{d^{3} P_{h}} & =\frac{2 \alpha_{s}^{2} M_{h}}{s^{2}} \varepsilon^{P_{h} p p^{\prime} S_{\perp}} \int \frac{d x}{x} f_{1}(x) \int \frac{d x^{\prime}}{x^{\prime}} f_{1}\left(x^{\prime}\right) \int \frac{d z}{z^{3}} \delta(\hat{s}+\hat{t}+\hat{u}) \times \\
& \times\left[\frac{D_{T}(z)}{z} \hat{\sigma}_{1}-\left\{\frac{d}{d(1 / z)} \frac{D_{1 T}^{\perp(1)}(z)}{z}\right\} \hat{\sigma}_{2}-D_{1 T}^{\perp(1)}(z) \hat{\sigma}_{3}+\right. \\
& \left.+\int_{z}^{\infty} \frac{d z_{1}}{z_{1}^{2}}\left(\frac{z_{1}}{1 / z-1 / z_{1}}\right)\left(\operatorname{Im} \widehat{D}_{F T}\left(z, z_{1}\right)+\operatorname{Im} \widehat{G}_{F T}\left(z, z_{1}\right)\right) \hat{\sigma}_{4}\right],
\end{aligned}
$$

where $s=\left(p+p^{\prime}\right)^{2}$ is the center of mass energy squared, $f_{1}(x)$ is the unpolarized twist-2 Parton Distribution Function (PDF) in the nucleon, $\hat{\sigma}_{1,2,3,4}$ are the partonic cross sections, and summation over all channels and the parton types is implied. For actual forms of $\hat{\sigma}_{1,2,3,4}$, see [11].

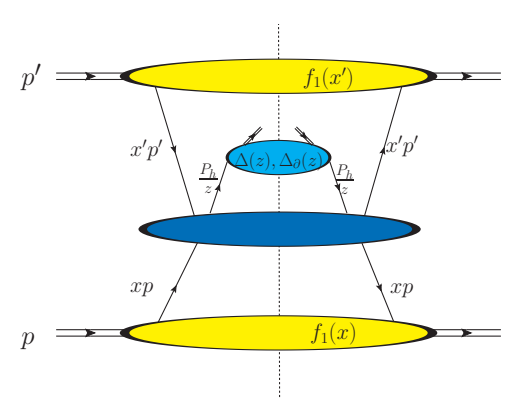

(a)

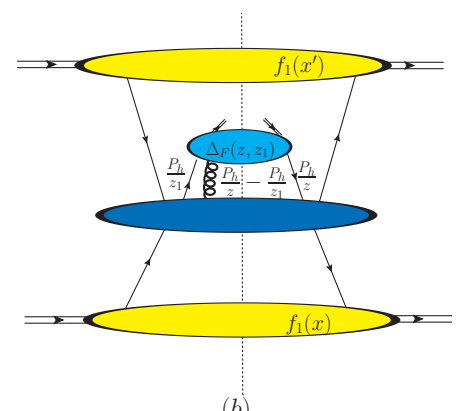

(b)

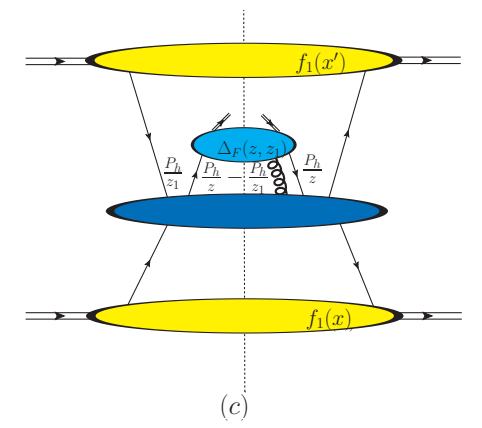

Figure 1: Generic diagrams giving rise to the twist-3 quark fragmentation contribution to the polarized cross section for the process (2.6). The top blob and bottom blob indicate the unpolarized twist-2 PDFs in the protons. The second blob from the top represents the twist-3 FFs for $\Lambda: \Delta(z)$ and $\Delta_{\partial}(z)$ in (a) and $\Delta_{F}\left(z, z_{1}\right)$ in (b) and (c). The second blob from the bottom is the partonic hard scattering parts.

\section{Contribution from Twist-3 gluon fragmentation functions}

\subsection{Twist-3 purely gluonic fragmentation functions for $\Lambda$}

In this section, we summarize the purely gluonic twist-3 Fragmentation Functions (FFs) [19]. (Our notation here is slightly different.) Similarly to the quark FFs, they are classified into three 
types. First, the gluon FFs defined from the 2-gluon correlators are given by

$$
\begin{aligned}
& \hat{\Gamma}^{\alpha \beta}(z)=\frac{1}{N^{2}-1} \sum_{X} \int \frac{d \lambda}{2 \pi} e^{-i \frac{\lambda}{z}}\left\langle 0\left|F_{a}^{\mathrm{w} \beta}(0)\right| h\left(P_{h}, S_{h}\right) X\right\rangle\left\langle h\left(P_{h}, S_{h}\right) X\left|F_{a}^{\mathrm{w} \alpha}(\lambda \mathrm{w})\right| 0\right\rangle= \\
& =-g_{\perp}^{\alpha \beta} G(z)-i M_{h} \varepsilon^{P_{h} \mathrm{w} \alpha \beta}\left(S_{h} \cdot \mathrm{w}\right) \Delta \hat{G}(z)-i M_{h} \varepsilon^{\alpha \beta S_{h \perp} \mathrm{w}} \Delta \hat{G}_{3 T}(z)+M_{h} \varepsilon^{P_{h} \mathrm{w} S_{h \perp}\{\alpha}{ }_{\mathrm{w}}^{\beta\}} \Delta \hat{G}_{3 \bar{T}}(z),
\end{aligned}
$$

where $\Delta G_{3 T}(z)$ and $\Delta G_{3 \bar{T}}(z)$ are the intrinsic twist-3 FFs, while the other two are twist-2 FFs. These functions are defined as real. Among these FFs, $\Delta G_{3 \bar{T}}(z)$ is naïvely $T$-odd, while the other three are naïvely $T$-even.

Second, we need the kinematical gluon FFs obtained from the derivative of the 2-gluon correlator. For a transversely polarized $\Lambda$, they are parametrized as

$$
\begin{aligned}
\hat{\Gamma}_{\partial}^{\alpha \beta \gamma}(z)= & \frac{1}{N^{2}-1} \sum_{X} \int \frac{d \lambda}{2 \pi} e^{-i \frac{\lambda}{z}}\left\langle 0\left|F_{a}^{\mathrm{w} \beta}(0)\right| h\left(P_{h}, S_{\perp}\right) X\right\rangle\left\langle h\left(P_{h}, S_{\perp}\right) X\left|F_{a}^{\mathrm{w} \alpha}(\lambda \mathrm{w})\right| 0\right\rangle \overleftarrow{\partial}^{\gamma}= \\
= & -i \frac{M_{h}}{2} g_{T}^{\alpha \beta} \varepsilon^{P_{h} \mathrm{w} S_{\perp} \gamma} \hat{G}_{T}^{(1)}(z)+\frac{M_{h}}{2} \varepsilon^{P_{h} \mathrm{w} \alpha \beta} S_{\perp}^{\gamma} \Delta \hat{G}_{T}^{(1)}(z) \\
& -i \frac{M_{h}}{8}\left(\varepsilon^{P_{h} \mathrm{w} S_{\perp}\{\alpha} g^{\beta\} \gamma}+\varepsilon^{P_{h} \mathrm{w} \gamma\{\alpha} S_{\perp}^{\beta\}}\right) \Delta \hat{H}_{T}^{(1)}(z),
\end{aligned}
$$

where each FF is defined to be real, and can also be written as the $k_{T}^{2} / M_{h}^{2}$-moments of the twist-2 transverse-momentum-dependent gluon FFs. Among the three FFs in 3.2$), \hat{G}_{T}^{(1)}(z)$ and $\Delta \hat{H}_{T}^{(1)}(z)$ are naïvely $T$-odd, while $\Delta \hat{G}_{T}^{(1)}(z)$ is naïvely $T$-even.

Finally, we introduce the dynamical twist-3 gluon FFs defined from the 3-gluon correlators. Presence of the two structure constants for the color SU(3) group, $d_{a b c}$ and $i f_{a b c}$, allows us to construct two types of FFs from three gluon field strength tensors. They are defined as

$$
\begin{aligned}
& \hat{\Gamma}_{F A}^{\alpha \beta \gamma}\left(\frac{1}{z_{1}}, \frac{1}{z_{2}}\right)= \\
& =\frac{-i f_{a b c}}{N^{2}-1} \sum_{X} \int \frac{d \lambda}{2 \pi} \int \frac{d \mu}{2 \pi} e^{-i \frac{\lambda}{z_{1}}} e^{-i \mu\left(\frac{1}{z_{2}}-\frac{1}{z_{1}}\right)}\left\langle 0\left|F_{b}^{\mathrm{w} \beta}(0)\right| h\left(P_{h}, S_{\perp}\right) X\right\rangle\left\langle h\left(P_{h}, S_{\perp}\right) X\left|F_{a}^{\mathrm{w} \alpha}(\lambda \mathrm{w}) g F_{c}^{\mathrm{w} \gamma}(\mu \mathrm{w})\right| 0\right\rangle= \\
& =\frac{M_{h}}{z_{2}}\left(N_{F 1}\left(\frac{1}{z_{1}}, \frac{1}{z_{2}}\right) g^{\alpha \gamma} \varepsilon^{P_{h} \mathrm{w} S_{\perp} \beta}+N_{F 2}\left(\frac{1}{z_{1}}, \frac{1}{z_{2}}\right) g^{\beta \gamma} \varepsilon^{P_{h} \mathrm{w} S_{\perp} \alpha}-N_{F 2}\left(\frac{1}{z_{2}}-\frac{1}{z_{1}}, \frac{1}{z_{2}}\right) g^{\alpha \beta} \varepsilon^{P_{h} \mathrm{w} S_{\perp} \gamma}\right) \\
& \hat{\Gamma}_{F S}^{\alpha \beta \gamma}\left(\frac{1}{z_{1}}, \frac{1}{z_{2}}\right)= \\
& =\frac{d_{a b c}}{N^{2}-1} \sum_{X} \int \frac{d \lambda}{2 \pi} \int \frac{d \mu}{2 \pi} e^{-i \frac{\lambda}{z_{1}}} e^{-i \mu\left(\frac{1}{z_{2}}-\frac{1}{z_{1}}\right)}\left\langle 0\left|F_{b}^{\mathrm{w} \beta}(0)\right| h\left(P_{h}, S_{\perp}\right) X\right\rangle\left\langle h\left(P_{h}, S_{\perp}\right) X\left|F_{a}^{\mathrm{w} \alpha}(\lambda \mathrm{w}) g F_{c}^{\mathrm{w} \gamma}(\mu \mathrm{w})\right| 0\right\rangle= \\
& =\frac{M_{h}}{z_{2}}\left(O_{F 1}\left(\frac{1}{z_{1}}, \frac{1}{z_{2}}\right) g^{\alpha \gamma} \varepsilon^{P_{h} \mathrm{w} S_{\perp} \beta}+O_{F 2}\left(\frac{1}{z_{1}}, \frac{1}{z_{2}}\right) g^{\beta \gamma} \varepsilon^{P_{h} \mathrm{w} S_{\perp} \alpha}+O_{F 2}\left(\frac{1}{z_{2}}-\frac{1}{z_{1}}, \frac{1}{z_{2}}\right) g^{\alpha \beta} \varepsilon^{P_{h} \mathrm{w} S_{\perp} \gamma}\right)
\end{aligned}
$$

The four functions $N_{F 1}, N_{F 2}, O_{F 1}$ and $O_{F 2}$ are complex and depend on two lightcone momentum fractions $z_{1}$ and $z_{2}$. Although the tensor decomposition in (3.3) and (3.4) is in parallel to the case of the three-gluon distribution functions [6], the number of independent FFs is four times as large as for the distribution functions. For the polarized hyperon production, the naïvely $T$-odd FFs $\Delta \hat{G}_{3 \bar{T}}$, $\hat{G}_{T}^{(1)}, \Delta \hat{H}_{T}^{(1)}, \operatorname{Im} N_{F i}$ and $\operatorname{Im} O_{F i}(i=1,2)$ contribute to the twist- 3 cross section. 


\subsection{Twist-3 gluon fragmentation contribution to the cross section}

The twist-3 gluon-fragmentation contribution to the process (2.6) is diagrammatically shown in Fig. 2. As in the case of the twist-3 quark-fragmentation contribution [11], the cross section arises as a nonpole contribution. Applying the collinear expansion in Feynman gauge developed in $[8,20]$, one can show that the corresponding twist- 3 cross section can be eventually expressed in terms of the gauge invariant correlation functions defined in the previous subsection as

$$
\begin{gathered}
E_{P_{h}} \frac{d \sigma\left(p, p^{\prime}, P_{h}, S_{\perp}\right)}{d^{3} \vec{P}_{h}}=\frac{1}{16 \pi^{2} s} \int \frac{d x}{x} f(x) \int \frac{d x^{\prime}}{x^{\prime}} f\left(x^{\prime}\right)\left[\Omega_{\alpha}^{\mu} \Omega_{\beta}^{v} \int d z \operatorname{Tr}\left[\hat{\Gamma}^{\alpha \beta}(z) S_{\mu v}\left(P_{h} / z\right)\right]-\right. \\
-i \Omega_{\alpha}^{\mu} \Omega_{\beta}^{v} \Omega_{\gamma}^{\lambda} \int d z \operatorname{Tr}\left[\left.\hat{\Gamma}_{\partial}^{\alpha \beta \gamma}(z) \frac{\partial S_{\mu v}(k)}{\partial k^{\lambda}}\right|_{c . l .}\right]+\operatorname{Re}\left\{-i \Omega_{\alpha}^{\mu} \Omega_{\beta}^{v} \Omega_{\gamma}^{\lambda} \int \frac{d z}{z} \int \frac{d z_{1}}{z_{1}} P\left(\frac{1}{1 / z_{1}-1 / z}\right) \times\right. \\
\left.\left.\times \operatorname{Tr}\left[\left(\frac{i f^{a b c}}{N} \hat{\Gamma}_{F A}^{\alpha \beta \gamma}\left(\frac{1}{z_{1}}, \frac{1}{z}\right)+d^{a b c} \frac{N}{N^{2}-4} \hat{\Gamma}_{F S}^{\alpha \beta \gamma}\left(\frac{1}{z_{1}}, \frac{1}{z}\right)\right) S_{\mu v \lambda, a b c}^{L}\left(z_{1}, z\right)\right]\right\}\right],
\end{gathered}
$$

where $\Omega_{\beta}^{\alpha}=g_{\beta}^{\alpha}-P_{h}^{\alpha} \mathrm{w}_{\beta} . S_{\mu v}(k)$ and $S_{\mu \nu \lambda, a b c}^{L}\left(z_{1}, z\right)$ are the partonic hard scattering parts corresponding to the fragmentation matrix elements given in Eqs. 3.1)- 3.4). Based on this formula, we have derived the LO partonic hard cross sections. The derivation of the above formula and the partonic hard cross sections for each FF in each channel will be reported elsewhere.

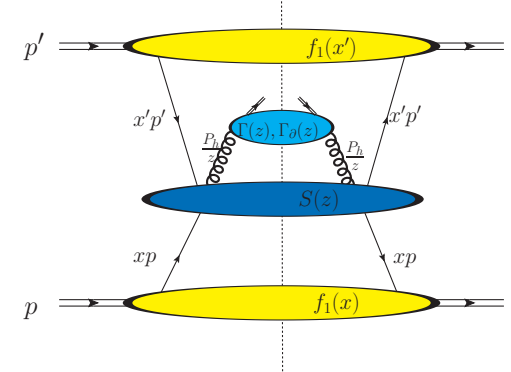

(a)

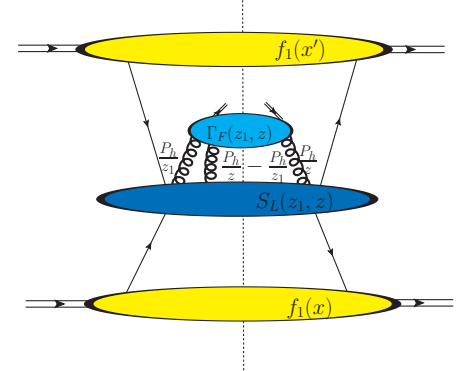

$(b)$

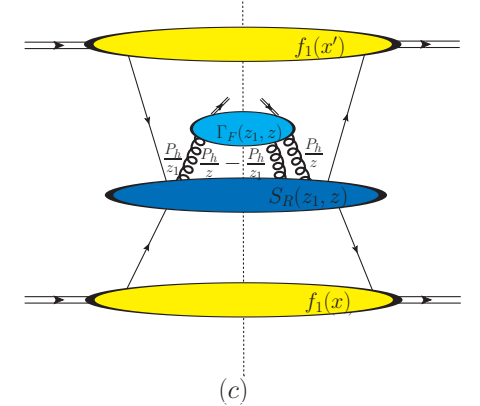

(c)

Figure 2: Generic diagrams giving rise to the twist-3 fragmentation contribution to the polarized cross section for the process (2.6). The top blob and bottom blob indicate the unpolarized twist-2 PDFs in the protons. The second blob from the top represents the fragmentation matrix elements for $\Lambda: \hat{\Gamma}(z)$ and $\hat{\Gamma}_{\partial}(z)$ in (a) and $\hat{\Gamma}_{F}\left(z_{1}, z\right)$ in (b). The second blob from the bottom is the partonic hard scattering parts: $S(z)$ in (a) and $S_{\alpha, a b c}^{L}\left(z_{1}, z\right)$ in (b) and (c).

\section{Summary}

In this work we studied the twist-3 fragmentation contribution to the polarized hyperon production in unpolarized proton-proton collision, $p p \rightarrow \Lambda^{\uparrow} X$, in the collinear twist-3 factorization. For the twist-3 quark fragmentation contribution, we have presented the complete LO cross section. The e.o.m. relation and the LIR among the twist-3 FFs have lead to the frame-independent twist-3 cross section. For the twist-3 gluon fragmentation contribution, we have formulated the method of calculating the cross section in terms of the complete set of the gluon FFs. Details of the calculation will be reported elsewhere. 


\section{Acknowledgments}

The work of K. Yabe and Y. Koike is supported by the Grant-in-Aid for Scientific Research from JSPS under Contract Nos. 18J11148 and 26287040. The work of A.Metz and D.Pitonyak is supported by the U.S. Department of Energy, Office of Science, Office of Nuclear Physics, within the framework of the TMD Topical Collaboration. The work of A.Metz is also supported by the National Science Foundation under grant number PHY-1516088. The work of S. Yoshida is supported by the U.S. Department of Energy, Office of Science under Contract No. DE-AC5206NA25396, the LANL LDRD Program, NSFC of China under Project No. 11435004 and research startup funding at SCNU.

\section{References}

[1] G. Bunce et al., Phys. Rev. Lett. 36 (1976) 1113.

[2] A. V. Efremov and O. V. Teryaev, Sov. J. Nucl. Phys. 36 (1982) 140 [Yad. Fiz. 36 (1982) 242].

[3] J. w. Qiu and G. F. Sterman, Phys. Rev. Lett. 67 (1991) 2264; Phys. Rev. D 59 (1999) 014004 [hep-ph/9806356].

[4] C. Kouvaris, J. W. Qiu, W. Vogelsang and F. Yuan, Phys. Rev. D 74 (2006) 114013 [hep-ph/0609238].

[5] H. Eguchi, Y. Koike and K. Tanaka, Nucl. Phys. B 763 (2007) 198 [hep-ph/0610314].

[6] H. Beppu, Y. Koike, K. Tanaka and S. Yoshida, Phys. Rev. D 82 (2010) 054005 [arXiv:1007.2034 [hep-ph]].

[7] A. Metz and D. Pitonyak, Phys. Lett. B 723, 365 (2013) Erratum: Phys. Lett. B 762 (2016) 549 [arXiv:1212.5037 [hep-ph]].

[8] K. Kanazawa and Y. Koike, Phys. Rev. D 88 (2013) 074022 [arXiv:1309.1215 [hep-ph]].

[9] K. Kanazawa, Y. Koike, A. Metz and D. Pitonyak, Phys. Rev. D 89 (2014) 111501(R) [arXiv:1404.1033 [hep-ph]].

[10] L. Gamberg, Z. B. Kang, D. Pitonyak and A. Prokudin, Phys. Lett. B 770 (2017) 242 [arXiv:1701.09170 [hep-ph]].

[11] Y. Koike, A. Metz, D. Pitonyak, K. Yabe and S. Yoshida, Phys. Rev. D 95 (2017) 114013 [arXiv:1703.09399 [hep-ph]].

[12] Y. Kanazawa and Y. Koike, Phys. Rev. D 64 (2001) 034019 [hep-ph/0012225].

[13] J. Zhou, F. Yuan and Z. T. Liang, Phys. Rev. D 78 (2008) 114008 [arXiv:0808.3629 [hep-ph]].

[14] Y. Koike, K. Yabe and S. Yoshida, Phys. Rev. D 92 (2015) 094011 [arXiv:1509.06830 [hep-ph]].

[15] K. Kanazawa, Y. Koike, A. Metz, D. Pitonyak and M. Schlegel, Phys. Rev. D 93 (2016) 054024 [arXiv:1512.07233 [hep-ph]].

[16] P. J. Mulders and R. D. Tangerman, Nucl. Phys. B 461 (1996) 197 Erratum: Nucl. Phys. B 484 (1997) 538 [hep-ph/9510301].

[17] A. Bacchetta, M. Diehl, K. Goeke, A. Metz, P. J. Mulders and M. Schlegel, JHEP 0702 (2007) 093 [hep-ph/0611265].

[18] P. J. Mulders and J. Rodrigues, Phys. Rev. D 63 (2001) 094021 [hep-ph/0009343].

[19] L. Gamberg, Z. B. Kang, D. Pitonyak, M. Schlegel and S. Yoshida, JHEP 1901 (2019) 111 [arXiv: 1810.08645 [hep-ph]].

[20] Y. Hatta, K. Kanazawa and S. Yoshida, Phys. Rev. D 88 (2013) 014037 [arXiv:1305.7001 [hep-ph]]. 